verzeichnisses zur GOÄ mit dem 1,0-Fachen und die übrigen Leistungen des Gebührenverzeichnisses zur GOÄ mit dem 1,2-Fachen des Gebührensatzes der GOÄ vergütet.

\section{MMW Kommenta}

Es ist zwar ärgerlich, dass beim Basistarif nunmehr Konditionen vereinbart wurden, die unterhalb denen des Standardtarifs angesiedelt sind, zumal dort - im Gegensatz zum Standardtarif - eine Be- handlungspflicht für Vertragsärzte besteht. Angesichts der Tatsache, dass bisher aber nur knapp 15000 Bundesbürger in diesem Tarif versichert sind, kann das Ergebnis akzeptiert werden, zumal für den Fall, dass die Anzahl der im PKV-Basistarif versicherten Personen die Anzahl von 100 ooo Versicherten überschreitet, ein Sonderkündigungsrecht ohne Befristung vereinbart wurde. Entsprechend den gesetzlichen Vorgaben bewegen sich die Honorare auf EBM-Niveau. So bringt ein
Check-up im Basistarif ein Honorar von 30,78 Euro und im EBM von 30,98 Euro. Es gibt aber auch Unterschiede: Trotz des niedrigen Labormultiplikators dürften sich z.B. Praxislaborleistungen rechnen. So liegt das Honorar für eine BSG im Basistarif bei 3,15 Euro, im EBM bei o,25 Euro. Auch technische Leistungen schneiden z.T. etwas besser ab: Ein Belastungs-EKG nach Nr. 652 GOÄ ergibt im Basistarif ein Honorar von 31,12 Euro, im EBM z.B. nach Nr. 03321 von 19,78 Euro.

\title{
Waren die Massenimpfungen gegen die „Schweinegrippe“ notwendig?
}

Die Europäische Kommission wurde mittlerweile in einer mündlichen Anfrage aufgefordert, das bisherige Management im Umgang mit dem $\mathrm{H}_{1} \mathrm{~N}_{1}$ Grippevirus kritisch zu untersuchen. So sollen u.a. mögliche Interessenkonflikte zwischen der Europäischen Arzneimittelagentur EMEA, dem Europäischen Zentrum für die Prävention und Kontrolle von Krankheiten und Pharmafirmen untersucht werden, weil bereits seit Sommers 2009 bekannt gewesen sei, dass der $\mathrm{H}_{1} \mathrm{~N}_{1}$ Grippevirus weniger bedrohlich war als angenommen.

Auch die WHO gerät wegen ihres Umgangs mit der Schweinegrippe zunehmend in die Kritik. Im Europarat warfen Experten und Abgeordnete der Organisation vor, die Schweinegrippe voreilig zur Pandemie erklärt zu haben. Auch hier soll ein Untersuchungsausschuss klären, wie stark Wissenschaftler und staatliche Behör- den durch die Pharmaindustrie beeinflusst worden sind.

Die geplanten Initiativen sind zweifelsohne zu begrüßen. Nach momentaner Sachlage drängt sich der Verdacht auf, dass Millionen gesunder Menschen unnötigerweise dem Risiko mangelhaft getesteter Impfstoffe ausgesetzt wurden, nur weil die Definition für eine Pandemie von der WHO im vergangenen Mai geändert wurde und so die vergleichsweise harmlose Schweinegrippe zur Pandemie „hochgespielt" worden ist.

In Deutschland ist die aus dieser Fehlentscheidung resultierende finanzielle Belastung für die ohnehin in dieser Hinsicht gebeutelten Kassen im Moment noch unabsehbar. So wurden seit Beginn der Impfung gegen die Neue Influenza bis Ende Dezember 2009 allein in Berlin 127575 Impfdosen verimpft, insgesamt aber 245930 Impfdosen ausgeliefert und be- reits 10717 Impfdosen verworfen. Diesen Verlust zahlen zwar nicht die Kassen, aber die einzelnen Bundesländer aus Steuergeldern und damit wir alle.



Eine Pandemie, die keine war? 